\title{
Impact of using Mobile Sink on Hierarchical Routing Protocols for Wireless Sensor Networks
}

\author{
Ahmed Salim ${ }^{1}$, Asmaa Ahmed Badran ${ }^{2}$ \\ ${ }^{1}$ Math. Dept., Fac. of Sci., Univ. of Zagazig, Zagazig, P. O. Box 44519, Egypt \\ Math. Dept. \\ Fac. of Sci. and Arts, Al-mithnab, Qassim Univ., P. O. Box 931, Buridah 51931, \\ Al-mithnab, Kingdom of Saudi Arabia \\ ${ }^{2}$ Math. Dept. Univ. of Zagazig, Zagazig, Egypt \\ Ia.salim@zu.edu.eg, ${ }^{2}$ asmaa.ahmedbadran@gmail.com
}

\begin{abstract}
Wireless sensor networks (WSNs) are composed of many low cost, low power devices with sensing, local processing and wireless communication capabilities. In these networks energy constraint is very important. Routing protocols form one of the most important communication paradigms that greatly affect the performance of the wireless sensor networks. In this paper, we compare the performances of six hierarchical based routing protocols when mobile sink is used. We perform analytical simulations in terms of network lifetime and average energy consumption.
\end{abstract}

Keywords: wireless sensor networks, hierarchical routing protocols, static sink, mobile sink

\section{Introduction}

WSNs are consisting of a large number of sensor nodes that transform physical data into a form that would make it easier for the user to understand [1]. The naive approach to collect data from sensor nodes is direct one where each sensor node transmits the data directly to the sink which is located far away. The cost of data transmission from each sensor node to the sink is very high, thus nodes die quickly and hence reducing the lifetime of the network. Therefore, use a few transmissions as possible leads to efficient energy utilization. Routing protocols from one of the most important communication paradigms that greatly affect the performance of the wireless sensor networks; so that designing routing protocols for sensor networks is a vital aspect. In earlier research, static sink is used to gather data in WSNs.

However, if the sink is able to move, sensors can communicate with the sink when it is near to them, and so hop by hop delivery path of data becomes shorter. Mobile sink even can move close to nodes when the nodes' power is reduced. So the set of nodes near the mobile sink can change, which provide more balance of energy and as a result, network lifetime can increase [2]. The use of mobile sink in WSNs introduces some complications, such as detecting and tracking sink location to ensuring reliable data transfer between sensor nodes and the mobiles sink, and managing sensor nodes to support sink mobility $[3,17]$.

In this paper, our goal is to adding the concept of sink mobility in the hierarchical chain based routing protocols, like, PEGASIS [5], PEGASIS-E [8], COSEN [10], ECBSN [11], IECBSN [12], and EAPHRN [13], and then compare the performance of these protocols in terms of network life time and average energy consumption. The rest of the paper is organized as follows: Section 2 briefly reviews related work. In Section 3, we introduce 
our problem statement. The experimental simulation is presented in Section 4. In Section 5 , we conclude our work.

\section{Related Research}

Routing protocol for WSNs is defined as the way of data dissemination from the network field to the sink. Based on the network topology, there two type of routing protocols in WSNs, flat routing protocols and hierarchical routing protocols [20]. In flatbased routing protocol, sensor nodes have the same role and collaborate together to perform the sensing task and multi-hop communication. Since the flat routing is based on flooding, it has several demerits, such as large routing overhead and high energy dissipation.

In hierarchical-based routing protocols, the network is divided into several logical groups within a fixed area. The logical groups are called clusters. Sensor nodes collect the information in a cluster and a head node aggregates the information. Each sensor node delivers the sensing data to the head node in the cluster and the head node delivers the aggregated data to the sink which is located outside of the sensor network. Contrary to flat routing protocols, only a head node aggregates the collected information and sends it to the sink. Due to these advantages, sensor nodes can remarkably save their own energy.

In general, introducing hierarchical routing into the network's topology reduces number of transmissions in the network. It also provides energy efficiency as cluster heads aggregate the data's from its cluster members, thereby reduce duplication of transmission and enhance the network lifetime. Many researches in the last few years have explored hierarchical routing in WSN from different perspectives. A variety of protocols have been proposed for prolonging the life of WSN and for routing data to the sink. LEACH [4] is the first and most popular energy-efficient hierarchical clustering algorithm for WSNs that was proposed for reducing power consumption. PEGASIS (Power-Efficient gathering in Sensor Information Systems) [5], introduces only a routing protocol that is near optimal for a data-gathering problem in WSNs. The main idea of the PEGASIS protocol is the formation of a chain among the sensor nodes so that each node will receive from and transmit to a close neighbor. Gathered data moves from node to node, get fused, and eventually a designated node transmits it to the sink. The work in [6] proposes an efficient routing protocol called CCBRP (Chain-Chain based routing protocol). CCBRP protocol divides a WSN into a number of chains (Greedy algorithm is used to form each chain as in PEGASIS protocol) and runs in two phases. In the first phase, sensor nodes in each chain transmit data to their chain leader nodes in parallel. In the second phase, all chain leader nodes form a chain (also, using Greedy algorithm) and choose randomly a leader node then all chain leader nodes send their data to this chosen leader node. The chosen leader node fuses the data and forwards it to the sink. CCM (Chain-Cluster based mixed routing) protocol [7] makes full use of the advantages of LEACH and PEGASIS, and provides improved performance over both of them. CCM protocol mainly divides a WSN into a number of chains and runs in two phases. In the first phase, sensor nodes in each chain transmit data to their own chain head nodes in parallel, using an improved chain routing protocol. In the second phase, all chain head nodes grouped as a cluster in a selforganized manner, where they transmit fused data to a voted cluster head using the cluster based routing. In [8], the authors proposed an improved energy efficient PEGASIS based protocol (PEGASIS-E). PEGASIS-E uses average distance among the sensor nodes as the criteria for chaining, thereby providing better performance in terms of energy dissipation and amount of information sent to the sink. The simulation results obtained show that PEGASIS-E gives an increase in the network lifetime as compared to PEGASIS. Y.Song [9] proposed an Energy-Efficient Chain-Based routing protocol (EECB) that is an improvement over PEGASIS. EECB uses distances between nodes and the base station and remaining energy levels of nodes to decide which node will be the leader that takes 
charge of transmitting data to the base station. In [10], the authors proposed a Chain Oriented Sensor Network (COSEN) protocol which is a two-layer protocol where a number of chains are formed in order to cover the whole region. COSEN reduce the latency associated with the original PEGASIS protocol by forming multiple smaller chains instead of a single longer chain. At first several small, fixed-length chains are constructed using the same distance-based greedy heuristic of the original PEGASIS protocol and a chain leader is selected in each chain based on the highest remaining energy. Leader nodes are selected for certain number of rounds. These chain leaders again form a higher-level chain and among those leaders one is chosen depending on the distance to the sink and remaining energy-level; to accumulate all data from the network and send that data to the sink at each round whereas other nodes only communicate with its neighbor in the chain.

Energy Efficient Chain Based Sensor Network (ECBSN) [11] overcomes several problems of PEGASIS. ECBSN overcomes the problem of excessive delay; instead of one long chain in the network numbers of short chains are formed. In ECBSN, every first node in a chain becomes a leader node. Thus, it ignores the suitable proportion of nodes energy and distance between node and base station which optimize the leader selection according to the various application environments. ECBSN protocol has certain deficiencies like the non optimal selection of leader nodes in rounds, aggregation and transmission of data by head nodes that leads to unbalanced energy consumption [12]. An improved ECBSN protocol called IECBSN is proposed in [12]. IECBSN adopts a new method of selection of leader nodes based on selection value (SV) parameter, which outperforms ECBSN. EAPHRN (Energy Aware PEGASIS Based Hierarchical Routing) [13] is a chain based routing protocol in which node does not connects to the next closest node but connects to a random node that is not far than the Distance Threshold (DT). EAPHRN is divided into two phases, Chain Setup phase and Leader election phase. MobiRoute routing protocol proposed a model with a path predictable mobile sink to improve the packet deliver ratio. The sink is located at any point of network and the pause time of sink is longer than movement time. It has enough time to gather the data from nearby nodes [14]. In [15] Rakhshan et al. also focused on maximizing the network lifetime of WSNs by using sink mobility. Energy formula that determines the moving times of sink based on residual energy of sensor nodes is proposed in this work. Furthermore, approach to calculate optimum path is used, which results in increasing the network lifetime. In [16] Jafri et al. proposed mobility in multi-chain PEGASIS based protocol. In this work mobile sink is moved along its trajectory and stays for a sojourn time at sojourn location to guarantee complete data collection. This approach increased network lifetime to a distinct level, as compared to classical approaches. In [17], the authors develop an analytical model that describes the communication load distribution in a WSN. They prove that for network lifetime elongation, the best position for a static sink is the center of the circle when the WSN covers a circular area. The authors further demonstrate that using a mobile sink is beneficial, and the mobility trajectory should follow the periphery of the network.

The work in [19] is similar to our work, the authors compare five clustering routing protocols; Low Energy Adaptive Clustering Hierarchy (LEACH), Threshold Sensitive Energy Efficient Sensor Network (TEEN), Distributed Energy Efficient Clustering (DEEC) and two variants of TEEN which are Clustering and Multi-Hop Protocol in Threshold Sensitive Energy Efficient Sensor Network (CAMPTEEN) and Hierarchical Threshold Sensitive Energy Efficient Sensor Network (H-TEEN). They introduce the mobility of sink in the five proposed protocols and compare their performance. The main difference between our work and $[19,14,16,2]$ is that our 
work provides analytical simulations in terms of network lifetime and average energy consumption for different hierarchical chain based routing protocols.

\section{Problem Statement}

Our goal is to analyze the performance and to compare the hierarchical routing protocols such as PEGASIS, PEGASIS-E, COSEN, ECBSN, IECBSN, and EAPHRN by implementing the concept of sink mobility in them. We consider data collected by a single mobile sink travelling in the monitored region. Initially sink starts motion from the initial position of the bounded services area. Then the position of sink is varied at different locations of the network area according to different mobility scenarios (random, rectangle, circle and middle path).

In random mobility scenario, the sink initially is placed randomly on the edge of the area. Then a destination is randomly chosen and the sink moves towards this destination. Upon arrival, the sink pauses for a time and again chooses a new destination for the next movement. As a result, the sink is staying in a location for a certain period of time (pause time). While the sink is staying in a location for a certain period of time it broadcasts a start message to the network nodes. After receiving the start message each head node sends the data packets to the sink till receives the stop message. Before the sink changes its position, it broadcasts another message to reset the nodes and stop the transmission, to reduce the packet drop. After that sink changes to a new position and follow the same steps every time [18]. We use pause time because sink needs to collect the data packets before change its position and we have taken long pause time equals to round period time.

In rectangle mobility scenario, the sink moves along rectangle trajectory along the boundary of the network and pauses to collect data during round period then moves to next point. In middle path mobility scenario the sink moves back and forth along path at the middle of the network and pause for a period of time to collect data. In circle mobility scenario, the sink circles along the boundary of the network and pauses to collect data during round period then moves to next point in the next round.

\section{Experimental Simulation}

A simulator has been implemented using $\mathrm{C \#}$ to evaluate the performance of our proposed protocol. In our simulation, 100 sensor nodes are randomly deployed in a region of size $200 \mathrm{~m}$ x $200 \mathrm{~m}$ on a two dimensional plane and are uniformly distributed with sink (static and mobile). In order to measure the energy consumption of sensor nodes, we use the same energy parameters and radio model as discussed in [4], wherein energy consumption is mainly divided into two parts: receiving and transmitting messages. The transmission energy consumption needs additional energy to amplify the signal depending on the distance to the destination. Thus, to transmit a k-bits message a distance $\mathrm{d}$, the radio power consumption will be,

$$
E_{T x}(k, d)= \begin{cases}k E_{T x}+K \epsilon_{f s} d^{2} & d<d_{0} \\ k E_{T x}+K \epsilon_{f s} d^{4} & d<d_{0}\end{cases}
$$

and to receive this message, the radio expends will be

$$
E_{R x}(k)=k * E_{\text {elec }} .
$$

The simulated model parameters are given in Table 1. 
Table 1. Parameters used in the Simulation

\begin{tabular}{|c|c|}
\hline Parameter & Value \\
\hline Network Size & $200 \times 200$ \\
\hline Sink Location & $\begin{array}{l}\text { Fixed at }(50,50),(100,100) \text {, or }(50,300) \\
\text { Mobile (random, rectangle, circle or } \\
\text { middle path mobility scenario) }\end{array}$ \\
\hline Nodes & 100 \\
\hline Initial energy & 2 unit \\
\hline$E_{\text {elec }}$ & $50 \mathrm{~nJ} / \mathrm{bit}$ \\
\hline$\epsilon_{f s}$ & $10 \mathrm{pJ} / \mathrm{bit} / \mathrm{m}^{2}$ \\
\hline$\epsilon_{m p}$ & $0.00013 \mathrm{pJ} / \mathrm{bit} / \mathrm{m}^{4}$ \\
\hline$d_{0}$ & $87 m$ \\
\hline$E_{D A}$ & $5 \mathrm{~nJ} /$ bit/signal \\
\hline Data Packet size & 2000bits \\
\hline
\end{tabular}

In two different scenarios, Static Sink and Mobile Sink (random, rectangle, circle and middle path mobility scenario), we use the following performance metrics to indicate the performance of routing protocols:

- Average Energy Consumption: It is the average energy consumed by all the nodes in sending, receiving and forwarding operations.

The average energy consumption per round until the first node die can be estimated as:

$$
E_{\text {Average }}=\frac{\sum_{i=1}^{N} E_{i}}{r}
$$

Where $N$ is the number of sensor nodes in the considered WSN, and $\mathrm{r}$ is the number of rounds.

- Network Lifetime: It is the time interval from the start of operation (of the sensor network) until the death of the first alive node.

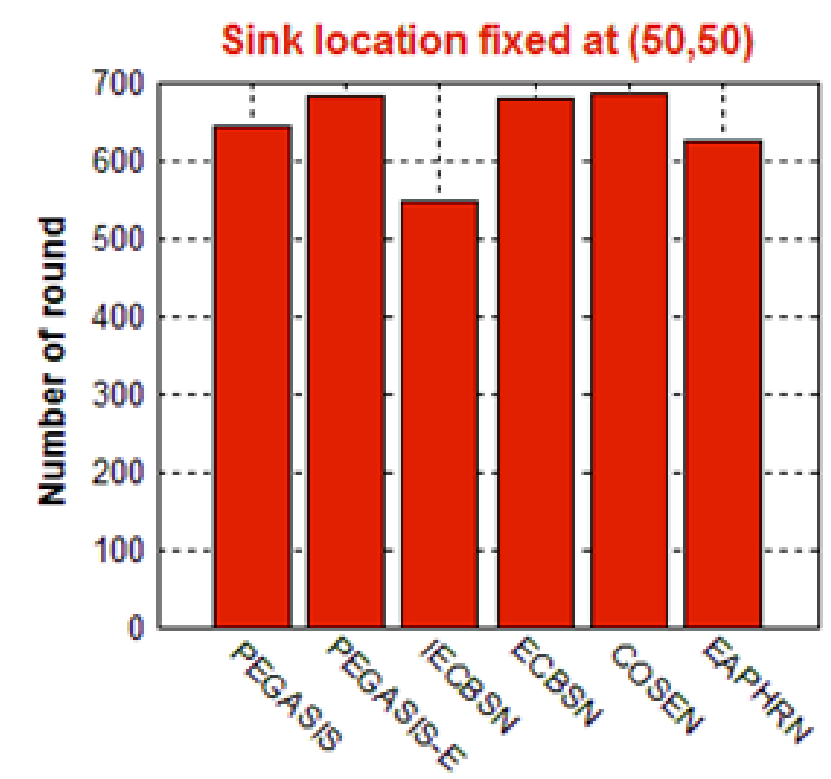

Figure 1. Network Lifetime for a 200x200 Network with Sink Locations at (50, 50) 


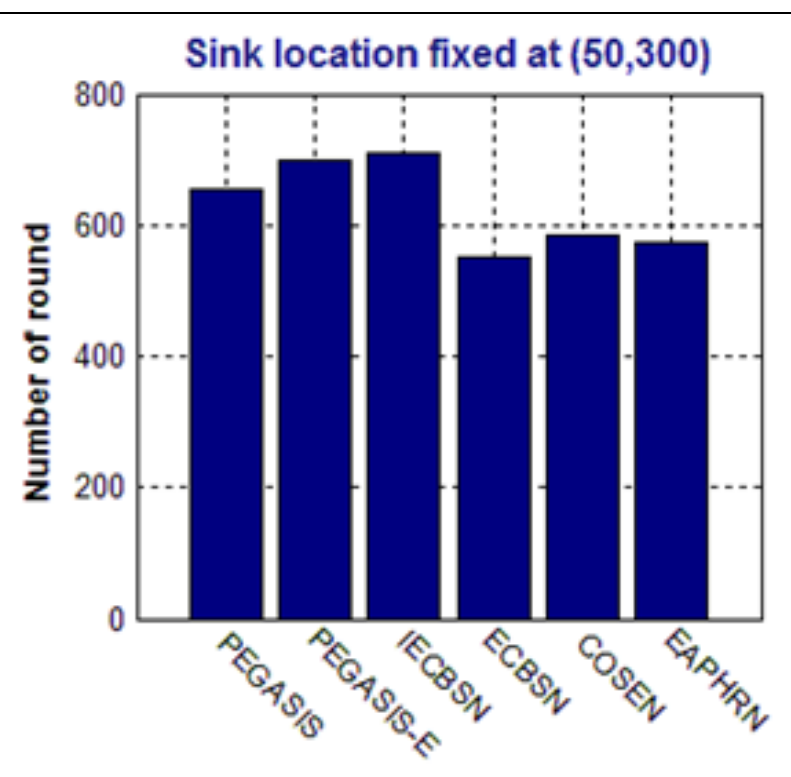

Figure 2. Network Lifetime for a 200x200 Network with Sink Locations at $(100,100)$

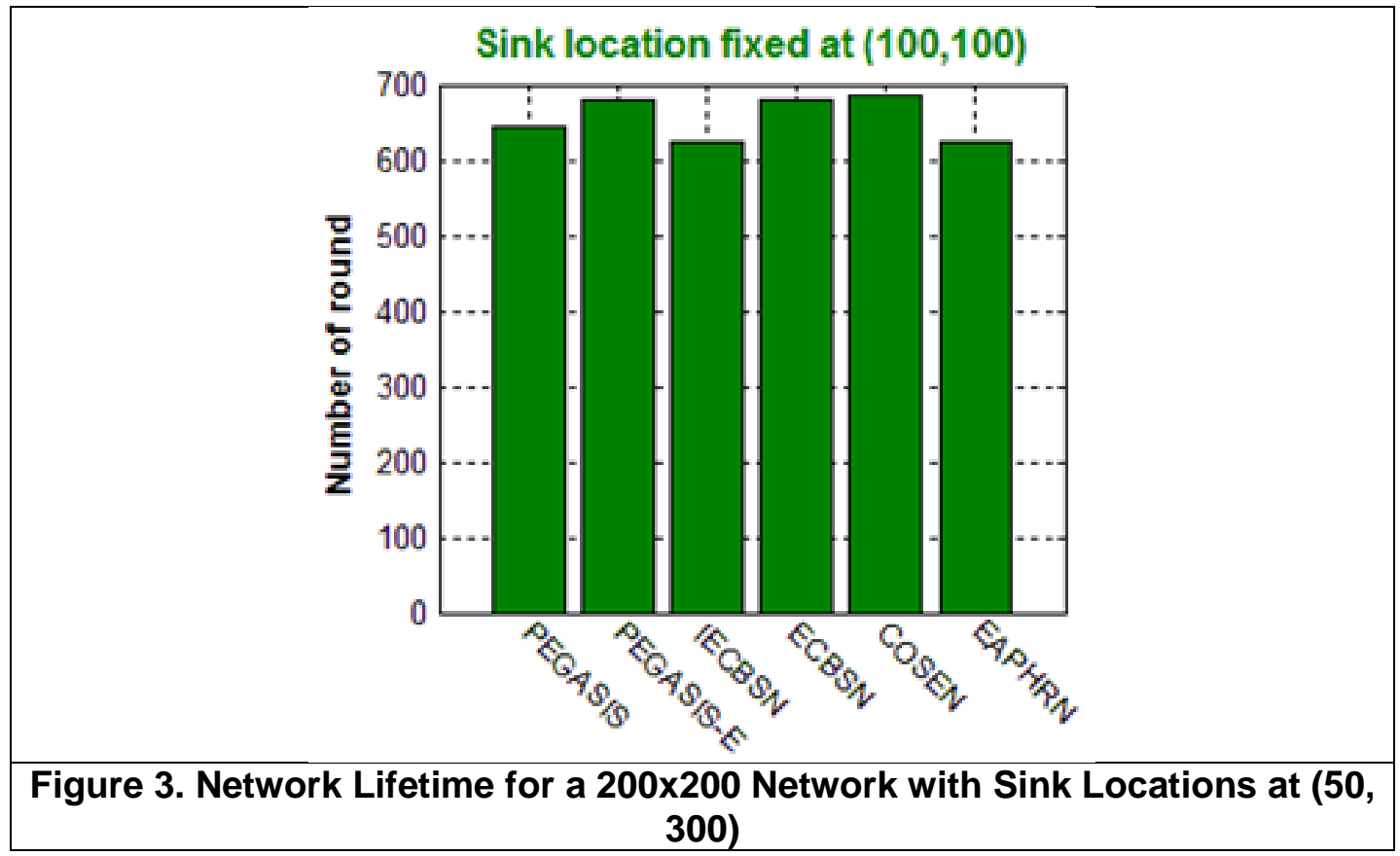

Figures 1, 2 and 3 indicate the lifetime for PEGASIS, PEGASIS-E, COSEN, ECBSN, IECBSN, and EAPHRN in case of static sink. We can see that the network lifetime for PEGASIS, PEGASIS-E, and IECBSN is increased with static sink at location $(50,300)$ and decreased when the sink is located near to the network center. However, the network lifetime for COSEN, ECBSN, and EAPHRN is decreased when the sink is located far away from the network center. 

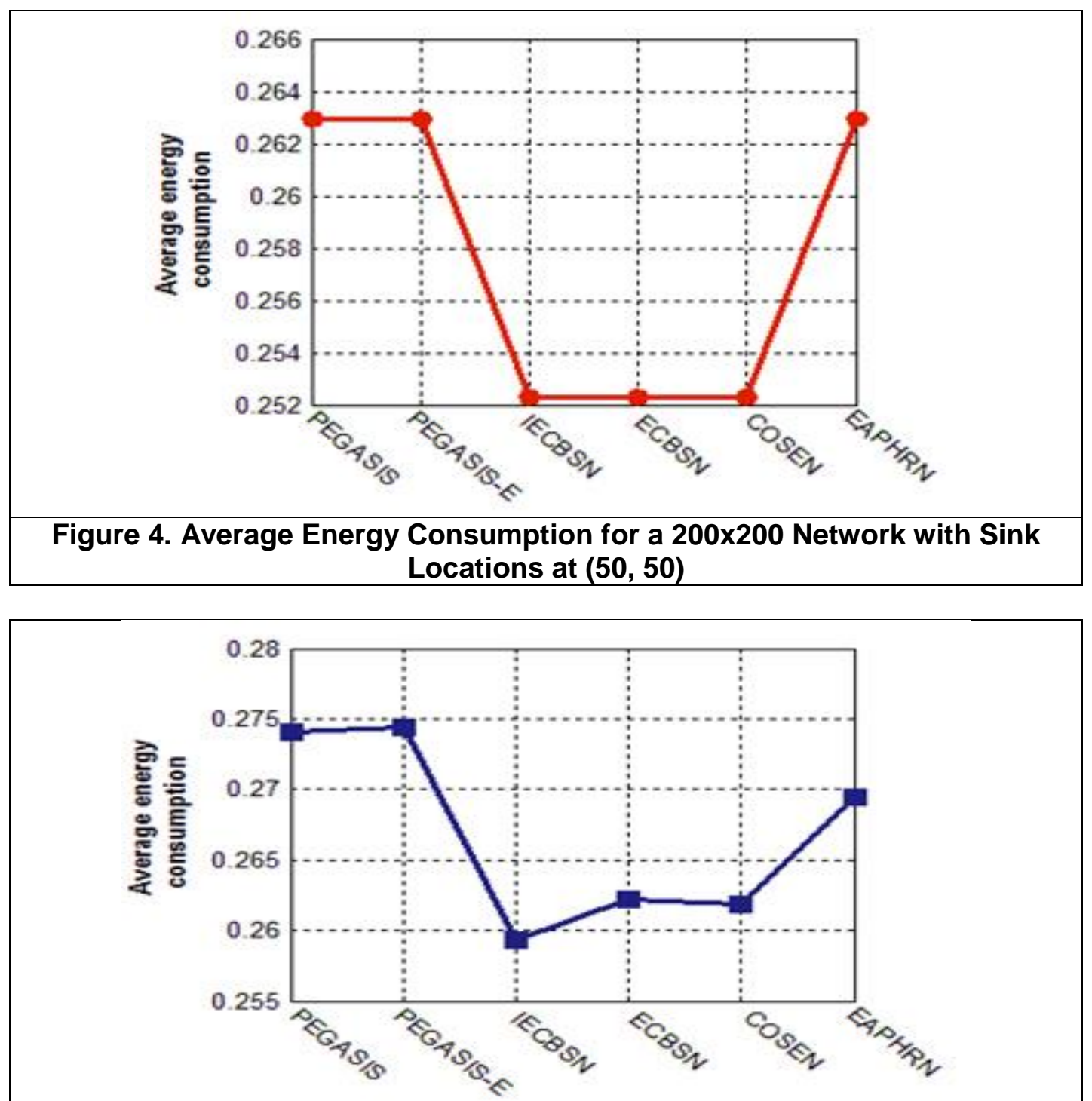

Figure 5. Average Energy Consumption for a 200x200 Network with Sink Locations at $(100,100)$

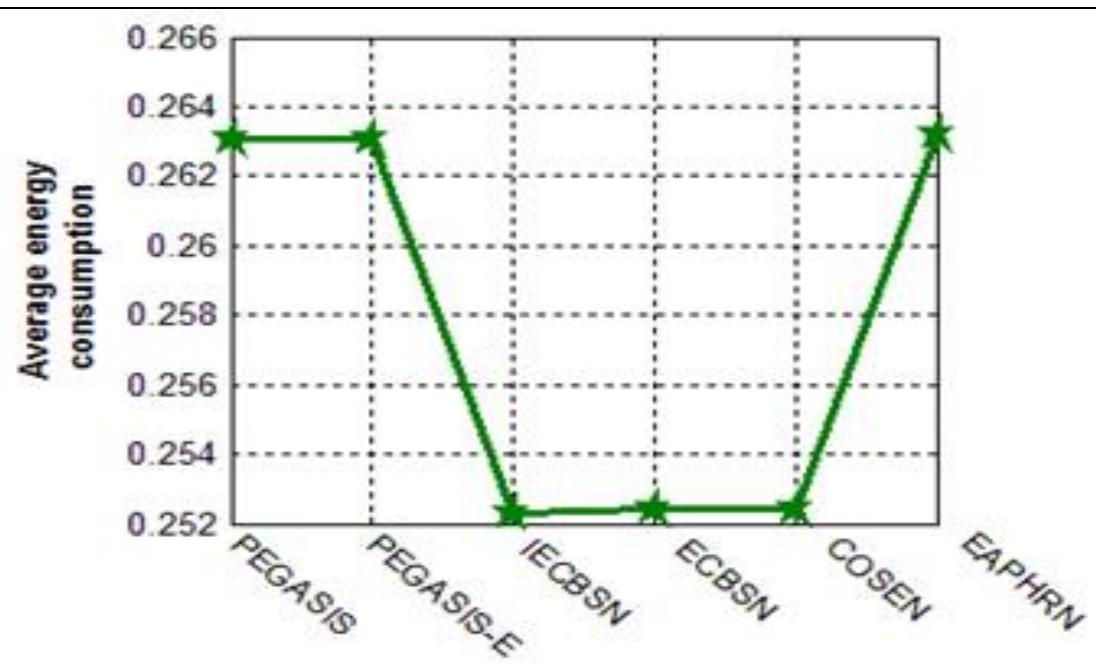

Figure 6. Average Energy Consumption for a 200x200 Network with Sink Locations at $(50,300)$ 
Figures 4, 5 and 6 indicate the average energy consumption for PEGASIS, PEGASISE, COSEN, ECBSN, IECBSN, and EAPHRN in case of static sink. We can see that the average energy consumption for all protocols is increased in case of sink location $(50,300)$ and decreased for sink locations $(50,50)$ and $(100,100)$; this is due to the increasing of the distance between the sink and the nodes implies increasing of consumed energy, which it can be observed from Equation 1.

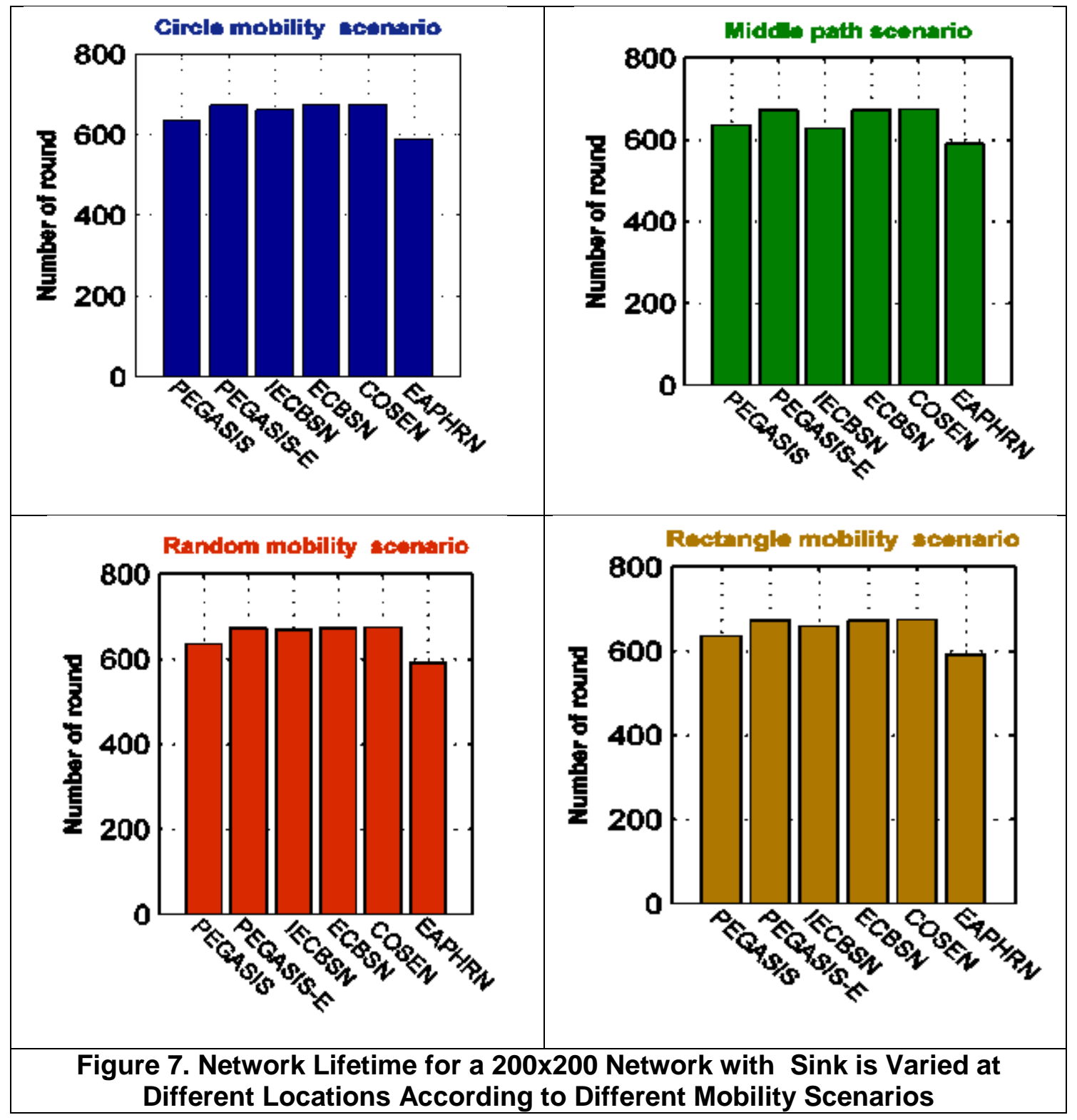

Figure 7 shows the lifetime for PEGASIS, PEGASIS-E, COSEN, ECBSN, IECBSN, and EAPHRN in case of mobile sink. We can observe that the network lifetime in PEGASIS, PEGASIS-E, COSEN, and ECBSN is decreased in all mobility scenarios. But on the other hand, we find that the network lifetime in IECBSN is increased in case of rectangle, middle path, and circle sink mobility scenarios compared to the static sink with locations $(50,50)$ and $(100,100)$. Furthermore, the network lifetime in IECBSN with random mobility scenario is decreased slightly compared to the static sink with location $(50,300)$. Only EAPHRN gives an increasing in the network lifetime in all mobility scenarios compared to its results in static sink. 


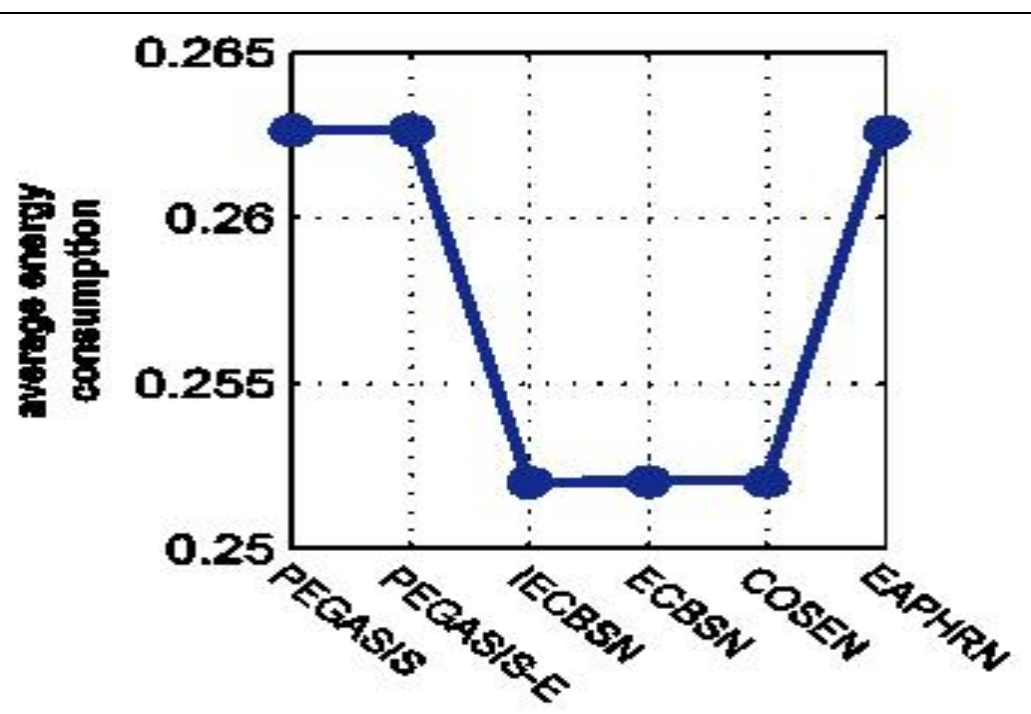

Figure 8. Average Energy Consumption for a 200x200 Network According to Circle Mobility Scenario

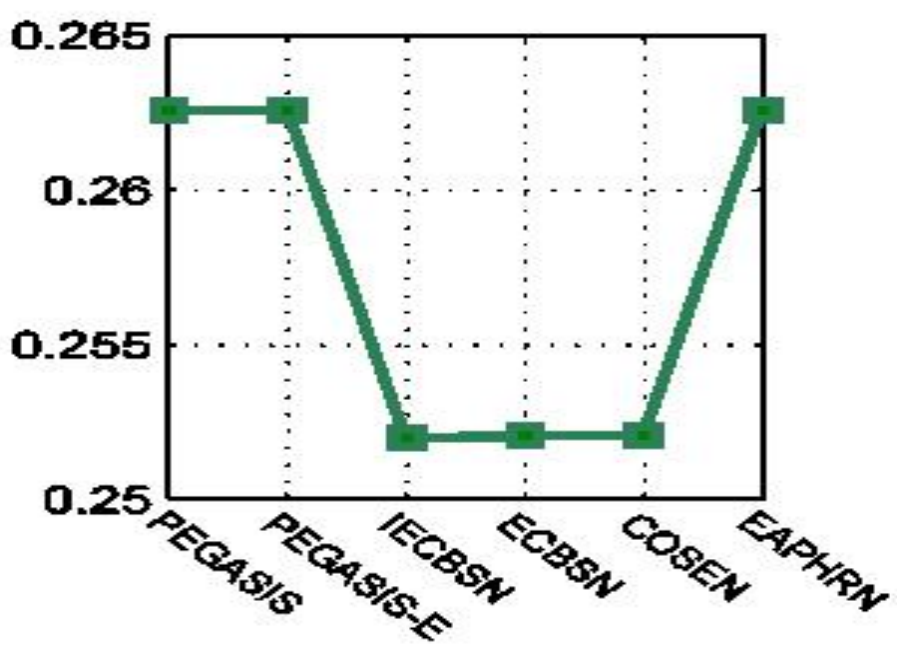

Figure 9. Average Energy Consumption for a 200x200 Network According to Middle Path Mobility Scenario

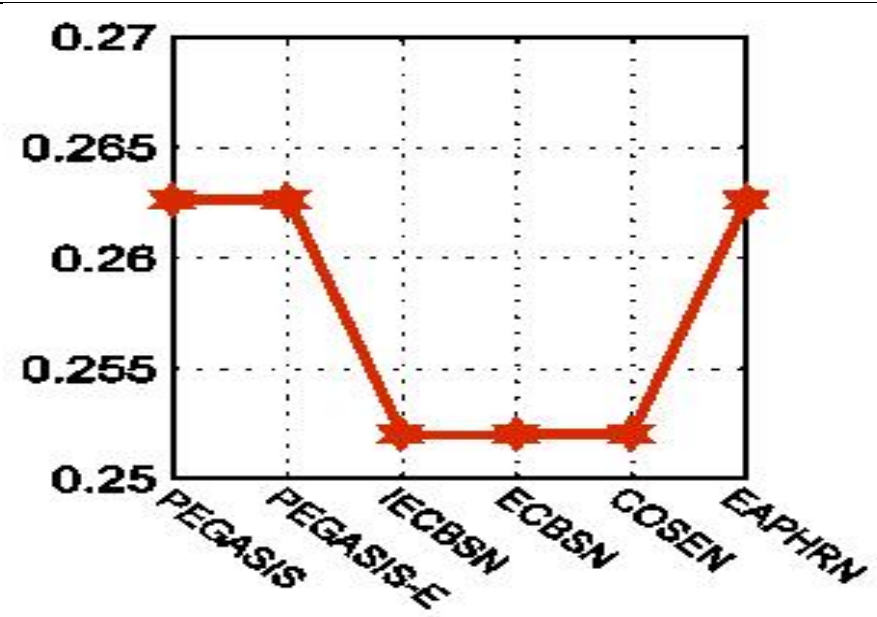

Figure 10. Average Energy Consumption for a 200x200 Network According to Random Mobility Scenario 


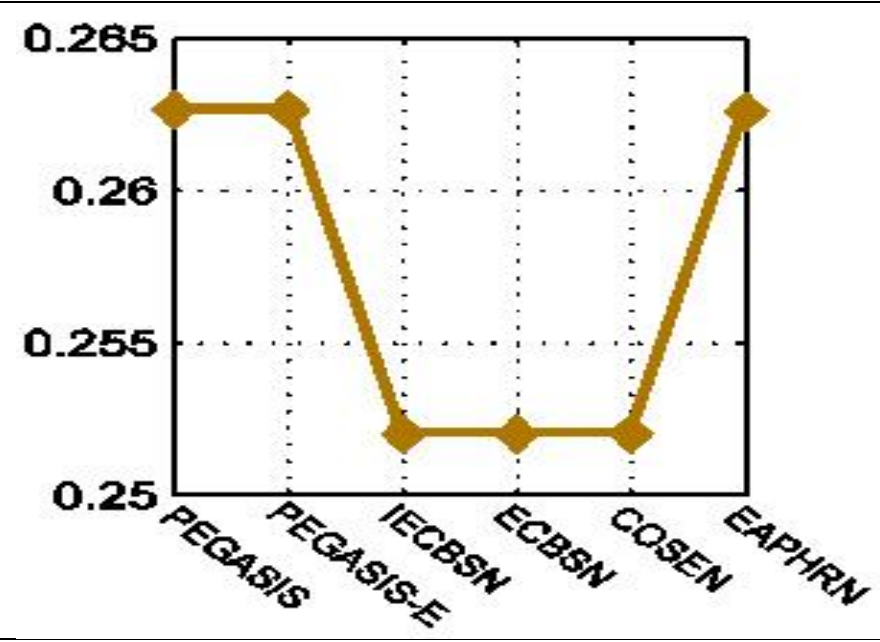

Figure 11. Average Energy Consumption for a 200x200 Network According to Rectangle Mobility Scenario

Figures 8, 9, 10 and 11 show the average energy consumption for PEGASIS, PEGASIS-E, COSEN, ECBSN, IECBSN, and EAPHRN in case of mobile sink according to different mobility scenarios. We can see that the average energy consumption in PEGASIS, PEGASIS-E, COSEN, ECBSN, IECBSN, and EAPHRN is decreased in all mobility scenarios.

By comparing network lifetime of all protocols in both cases, mobile sink and static sink, it is concluded that network lifetime of IECBSN and EAPHRN is increased in case of mobile sink. In addition to that the average consumed energy for all protocols is decreased in case of mobile sink. Only EAPHRN and IECBSN perform better in mobile sink and all other protocols give good results in static sink.

\section{Conclusion}

In this paper, we have introduced the mobility of sink in all proposed protocols, PEGASIS, PEGASIS-E, IECBN, ECBN, COSEN, and EAPHRN to compare their performances. We have introduced two scenarios are discussed to compare the performances of hierarchical routing protocols; in first scenario static sink is used and in the later one mobile sink is used. We have proposed different mobility scenarios, random, circle, middle path and rectangle mobility. The simulation results show that for various mobility scenarios EAPHRN and IECBSN perform better and all other protocols perform better in case of static sink. In our future work we aim to find the optimal path for sink movement and incorporate multiple sinks in the network.

\section{References}

[1] D. Estrin, R. Govindan, J. Heidemann and S. Kumar, "Next century challenges: scalable coordination in sensor networks", Proceedings of the 5th Annual ACM/IEEE International Conference on Mobile Computing and Networking, Seattle,Washington, USA, (1999), pp. 263-270.

[2] X. Min, S. Wei-ren, J. Chang-jiang and Z. Ying, "Energy efficient clustering algorithm for maximizing lifetime of wireless sensor networks", J AEU-International Journal of Electronics and Communications, vol. 64, (2010), pp. 289-298.

[3] Y. Faheem, S. Boudjit and K. Chen, "Data dissemination strategies in mobile sink Wireless Sensor Networks: A survey", Proceedings of the 2nd IFIP conference on Wireless days (WD'09), (2009).

[4] W. R. Heinzelman, A. Chandrakasan and H. Balakrishnan, "Energy-efficient communication protocol for wireless microsensor networks", Proceedings of the 33rd Hawaii International Conference on System Sciences, (2000), pp. 1-10.

[5] S. Lindsey and C. Raghavendra, "PEGASIS: Power Efficient gathering in sensor information systems", in Aerospace Conference Proceedings, vol. 3, (2002), pp. 1125-1130. 
[6] S. A. Ali and S. K. Refaay, "Chain-Chain Based Routing Protocol", IJCSI International Journal of Computer Science Issues, ISSN (Online): 1694-0814, vol. 8, Issue 3, no. 2, (2011) May.

[7] F. Tang, I. You, S. Guo, M. Guo and Y. Ma, "A chain-cluster based routing algorithm for wireless sensor networks", Journal of intelligent manufacturing, DOI: http://dx.doi.org/10.1007/s10845-0100413-4, vol. 23, no. 4, (2012), pp. 1305-1313.

[8] V. Nehra and A. K. Sharma, "PEGASIS-E: Power Efficient Gathering in Sensor Information System Extended", Global Journal of Computer Science and Technology Network, Web and Security, vol. 13, no. $15,(\mathbf{2 0 1 3})$

[9] Y. Yu and Y. Song, "An energy-efficient chain-based routing protocol in wireless sensor network", Computer Application and System Modeling (ICCASM), 2010 International Conference, IEEE, vol. 11, (2010), pp. 486-489.

[10] N. Abassum, Q. Ehsanul Kabir Haque Mamun, A. Ahsanul and Y. Urano, "A Chain Oriented Data Collection Protocol for Energy-Aware and Delay-Constrained WSN", African Journal of Information and Communication Technology, vol. 2, no. 3, (2006), pp. 11.

[11] S. Mahajan and J. Malhotra, "Enhanced Chain Technique Based Data Collection Sensor Network", International Journal of Computer Science, vol. 8, no. 3, (2011), pp. 83- 87.

[12] S. Mahajan, J. Malhotra and S. Sharma, "Improved Enhanced Chain Based Energy Efficient Wireless Sensor Network", Wireless Sensor Network, vol. 5, (2013), pp. 84-89.

[13] H. Alhasan, M. Qatawneh, A. Sleit and W. Almobaideen, "EAPHRN: energy-aware PEGASIS-based hierarchal routing protocol for wireless sensor networks", Journal of American Science, (2011).

[14] Y. Bi Y, L. Sun, J. Ma, N. Li, I. Khan and C. Chen, "Hums: An autonomous moving strategy in data gathering sensor networks", EURASIP Journal On Wireless Communication and Networking, vol. 15, (2007).

[15] A. Somasundara, A. Romamoorthy and M. Srivastava, "Mobile element scheduling for efficient data collection on wireless sensor", The 25th IEEE International Real- Time Systems Symposium, Los Angeles, CA, USA: IEEE, (2004) December 5-8, pp. 296-305.

[16] Y. Gu, D. Bozdag, E. Ekici, F. Ozguner and C. Lee, "Partitioning based mobile element scheduling in wireless sensor networks", The 2nd Annual IEEE Communications Society Conference on Sensor and Ad Hoc Communications and Networks, IEEE, (2005) September 26-29, pp. 386-395.

[17] J. Luo and J. P. Hubaux, "Joint mobility and routing for lifetime elongation in wireless sensor networks", 24th Annual Joint Conference of the IEEE Computer and Communications Societies, Ecole Polytech, de Lausanne, Switzerland: IEEE, (2005) March 13-17, pp. 1735-1746.

[18] D. Puthal, B. Sahoo and S. Sharma, "Dynamic Model for Efficient Data Collection in Wireless Sensor Networks with Mobile Sink", IJCST, vol. 3, no. 1, (2012) January-March.

[19] N. Javaid, Q. Ain, M. A. Khan, A. Javaid, Z. A. Khan and U. Qasim, "On Sink Mobility Trajectory in Clustering Routing Protocols in WSNs", CoRRabs/1304.0984, (2013).

[20] Z. Manap, B. M. Ali, C. K. Ng, N. K. Noordin and A. Sali, "A review on hierarchical routing protocols for wireless sensor networks", Wireless Personal Communications, vol. 72, no. 2, (2013), pp. 10771104. 
International Journal of Advanced Science and Technology Vol.77 (2015) 